\title{
Why dried blood spots are an ideal tool for CYP1A2 phenotyping.
}

Pieter M.M. De Kesel, Willy E. Lambert and Christophe P. Stove*

Laboratory of Toxicology, Department of Bioanalysis, Faculty of Pharmaceutical Sciences, Ghent University, Harelbekestraat 72, 9000 Ghent, Belgium

Accepted Manuscript Version De Kesel et al.

\section{Published in}

\section{Clinical Pharmacokinetics:}

\section{DOI 10.1007/s40262-014-0150-5}

*Corresponding author:

Christophe P. Stove

Laboratory of Toxicology

Department of Bioanalysis

Faculty of Pharmaceutical Sciences

Ghent University

Harelbekestraat 72

9000 Ghent

Belgium

E-mail: Christophe.Stove@UGent.be

tel.: +3292648121

fax: +3292648183 


\section{Abstract}

\section{Background and Objective}

Dried blood spot (DBS) sampling has gained wide interest in bioanalysis during the last decade. Also in pharmacokinetic and phenotyping studies, DBS-based sampling has already been successfully applied. However, all available phenotyping studies used small data sets and did not include a systematic evaluation of DBS-specific parameters. The latter is important since several of these factors still challenge the breakthrough of DBS in routine practice. In this study, caffeine and paraxanthine are determined in capillary DBS, venous DBS, whole blood and plasma for CYP1A2 phenotyping (CYP; cytochrome P450). The aim of this study was to explore the usefulness of DBS as a tool for CYP1A2 phenotyping.

\section{Methods}

A CYP1A2 phenotyping study was conducted in seventy-three healthy volunteers, who received a $150 \mathrm{mg}$ oral dose of caffeine. Six hours post-administration, caffeine and paraxanthine concentrations and paraxanthine:caffeine molar concentration ratios, i.e. the actual CYP1A2 phenotyping indices, were determined in capillary DBS (obtained by non-volumetric application, direct from the fingertip), venous DBS, whole blood and plasma. Furthermore, the impact of DBS-specific parameters, including hematocrit, volume spotted and punch location, was evaluated.

\section{Results}

Concentrations of caffeine and paraxanthine in capillary DBS were on average 12.7 respectively $13.8 \%$ lower than those in venous DBS and 31.5 respectively $33.1 \%$ lower than those in plasma. While these differences were statistically significant $(p<0.001)$, no significant difference was observed between the paraxanthine:caffeine molar ratios in the distinct evaluated matrices $(\mathrm{p} \geq$ 0.053). This ratio also alleviated the impact of hematocrit and volume spotted.

\section{Conclusions}

Using the largest DBS-based phenotyping study to date, we have demonstrated that CYP1A2 phenotyping in capillary DBS is a valid and convenient alternative for the classical plasma-based approach. Additionally, we have provided an objective basis as to why DBS are an ideal tool for CYP1A2 phenotyping. 


\section{Key points}

- A CYP1A2 phenotyping study, conducted in seventy-three healthy volunteers, demonstrated equivalence between phenotyping indices, i.e. paraxanthine:caffeine molar concentration ratios, in capillary DBS (obtained by non-volumetric application, direct from the fingertip) and venous plasma.

- While capillary DBS concentrations of caffeine and paraxanthine differed significantly from those in venous blood and plasma and were impacted by hematocrit and blood volume spotted, the paraxanthine:caffeine molar ratio essentially remained unaffected.

- Capillary DBS-based CYP1A2 phenotyping proved to be a valid and convenient alternative for the classical plasma-based approach. 


\section{Introduction}

During the last decade, dried blood spot (DBS) sampling has become a widely used microsampling technique in bioanalysis, owing to the development of more sensitive analytical techniques and various benefits offered by the technique itself [1-5]. Among the advantages are minimal invasiveness, easy sample collection and improved compound stability. This allows the collection of representative samples by an informed patient himself in his home-environment, followed by convenient and cost-effective transfer, e.g. via regular mail, to the analytical laboratory [6].

However, despite the many advantages, several issues concerning DBS analytics and the potential to fully replace venous sampling still remain. First of all, being a capillary sampling technique, the correlation between venous and capillary concentrations is one of the key points that needs to be evaluated when setting up DBS-based methods [7, 8]. Furthermore, adequate interpretation of DBS results requires comparison with plasma or serum concentrations, as clinical reference intervals are commonly based on the latter [9]. Finally, potential bias introduced by the spotting technique itself must be excluded, for example by comparing concentrations measured in venous whole blood samples with concentrations in venous DBS. From an analytical perspective, the (combined) influence of hematocrit (Hct), blood volume spotted and punch localization on a DBS result are important challenges to be addressed during method development and validation [2, 3, 10-12]. The degree of bias caused by these parameters needs to be evaluated on a case-by-case basis since it may be influenced by several factors, such as compound characteristics, filter paper type or extraction conditions.

CYP1A2 (CYP; cytochrome P450), an enzyme whose activity is subject to large inter-individual variability, is involved in the metabolism of several therapeutic drugs, including the antipsychotics clozapine and olanzapine. Consequently, the response to these drugs will be influenced by CYP1A2 status [13]. Since CYP1A2 activity is determined by genetic, environmental and nongenetic endogenous factors [14-16], phenotyping with a selective substrate rather than genotyping is commonly used to assess enzyme activity. Caffeine is widely accepted as model substrate of CYP1A2, owing to its safety profile and since the N3-demethylation to paraxanthine is almost uniquely controlled by CYP1A2. Measuring caffeine clearance via a full concentration-time profile is considered as the gold standard for CYP1A2 activity determination 
[17]. However, as this approach is inconvenient, the paraxanthine:caffeine ratio in plasma, measured 5-7h after controlled caffeine administration (100-200 mg), is a generally accepted metric for this purpose $[18,19]$.

A proof-of-principle of DBS-based phenotyping has already been shown for CYP3A4 [20] and CYP2C9 [21]. Very recently, two groups reported on the use of a cocktail approach to simultaneously phenotype multiple enzymes, including CYP1A2, in DBS [22, 23]. However, all these studies were conducted in a very limited number of volunteers $(\mathrm{n} \leq 16)$, hampering thorough statistical evaluation. In all cases, except for the study by Donzelli et al. [23], blood spots were volumetrically applied to the filter paper in a controlled environment [20-22]. Moreover, no systematic investigation of parameters potentially influencing the phenotyping index has been undertaken, yet. Therefore, the main objective of this study was to explore the usefulness of DBS as a matrix for CYP1A2 phenotyping.

\section{Methods}

\subsection{CYP1A2 phenotyping study}

A CYP1A2 phenotyping study was conducted in healthy volunteers. The study was approved by the Ethics Committee of Ghent University Hospital (B670201111655). Sample size was selected based on the following factors: i) feasibility of the study; ii) the possibility to conduct statistical analyses, such as Bland-Altman comparison and Passing-Bablok regression analysis; iii) estimation of the required sample size to investigate differences between caffeine and paraxanthine concentrations and paraxanthine:caffeine ratios at a significance level of 0.05 and power of $90 \%$. The latter calculation was based on data of a preliminary CYP1A2 phenotyping study in dried blood spots, whole blood and plasma [24]. Relevant differences between caffeine and paraxanthine concentrations in the different matrices were chosen at 0.08 and $0.04 \mu \mathrm{g} / \mathrm{mL}$ respectively, being $10 \%$ of the lowest concentrations measured in the preliminary study. For the paraxanthine:caffeine ratio, a difference of 0.05 was considered relevant. The highest required sample size for the intended comparisons between the different matrices resulting from this calculation was 59. Based on these data, a sample size of 73 individuals was considered adequate for the planned study. Written informed consent was obtained from each subject. Participants 
were asked to cease caffeine ingestion for 36 hours prior to intake of a capsule containing $150 \mathrm{mg}$ caffeine. Capillary DBS were collected 5 minutes before and 6 hours after administration. At the 6-hour time point ( \pm 5 minutes), venous whole blood samples were collected as well. Plasma samples and venous DBS were prepared within 1 hour after blood sampling. Concentrations of caffeine and paraxanthine (and the corresponding paraxanthine:caffeine molar concentration ratios) were determined in capillary DBS, venous DBS, whole blood and plasma.

\subsection{Sample collection}

Capillary DBS were obtained by direct application of a drop of blood from the fingertip onto Whatman 903 filter paper cards (WHA10334885, GE Healthcare, Dassel, Germany). Instructions on good sampling practices were given to all participants by means of a hands-on demonstration and an illustrated flyer. Following disinfection of the fingertip, finger pricks were made with an automated lancet (VAC366594, Becton Dickinson, Franklin Lakes, NJ, USA). The first drop of blood was wiped off and the following drops, at least four for every participant, were collected on the DBS cards, thereby ensuring that a single drop was used for every spot and avoiding direct contact between filter paper and fingertip. DBS of which both sides of the filter paper were colored were analyzed. Venous blood, collected in ethylenediaminetetraacetic acid (EDTA) tubes (Venosafe $^{\circledR} 4 \mathrm{~mL}$ VF-054SDK, Terumo, Leuven, Belgium), was used for preparing venous DBS by spotting $25 \mu \mathrm{L}$ of blood onto Whatman 903 filter paper using a calibrated pipette and for preparing plasma. Liquid samples were stored at $-20{ }^{\circ} \mathrm{C}$, DBS were dried for at least 2 hours and stored at ambient temperature in zip-closure plastic bags, containing two 5-g packets of desiccant (Minipax $^{\circledR}$ absorbent packets, Sigma-Aldrich, Diegem, Belgium), until analysis. For evaluating DBS-specific parameters, venous whole blood from a caffeine abstinent healthy, male volunteer was collected in EDTA tubes (Venosafe ${ }^{\circledR} 9 \mathrm{~mL}$ VF-109SDK, Terumo).

\subsection{Chemicals}

Caffeine, paraxanthine, theophylline, theobromine, the internal standards (IS) caffeine- ${ }^{13} \mathrm{C}_{3}$ and paraxanthine- ${ }^{13} \mathrm{C}_{4^{-}}{ }^{15} \mathrm{~N}_{3}$, and formic acid were purchased from Sigma-Aldrich. Methanol and acetonitrile, both of LC-MS quality, were from Biosolve (Valkenswaard, The Netherlands). A Synergy ${ }^{\circledR}$ Water Purification System (Merck Millipore, Overijse, Belgium) provided ultrapure water. 


\subsection{Sample preparation and analysis}

For all DBS analyses, 3-mm discs were punched from the center of a DBS using a Harris micropunch and cutting mat (Sigma-Aldrich). As capillary DBS were generated in a non-volumetric way, resulting in spots with varying sizes, we opted to punch 3-mm discs. These were extracted with $70 \mu \mathrm{L}$ of a methanol/water (80/20, v/v) mixture, containing $0.01 \%$ formic acid and the internal standards. For plasma and whole blood, we used a simple protein precipitation procedure, starting from $50 \mu \mathrm{L}$ sample, to which $10 \mu \mathrm{L}$ of IS solution was added, prior to the addition of $100 \mu \mathrm{L}$ methanol, containing $0.01 \%$ formic acid. After centrifugation, supernatants were diluted with water, containing $0.01 \%$ formic acid, until starting conditions were reached. Full details on sample preparation procedures are described elsewhere [24].

All samples were analyzed on a Waters Acquity UPLC ${ }^{\circledR}$ system (Waters, Milford, MA, USA) coupled to an $\mathrm{AB}$ SCIEX API $4000^{\mathrm{TM}}$ triple quadrupole mass spectrometer (AB SCIEX, Framingham, MA, USA), as described elsewhere [24]. Quantitative analyses were performed for caffeine and paraxanthine. Theophylline and theobromine, being metabolites of caffeine with the same molecular mass as paraxanthine, were qualitatively monitored in every analytical run to ensure selectivity. The methods used were fully validated based on U.S. Food and Drug Administration (FDA) and European Medicines Agency (EMA) guidelines for bioanalytical method validation [25, 26]. No interferences or carry-over were observed for any of the matrices. Linear calibration curves were obtained for both caffeine and paraxanthine (respectively 0.0510.0 and $0.025-5.0 \mu \mathrm{g} / \mathrm{ml})$. Intrabatch precision (\% CV; coefficient of variation) was below $11.40 \%$ (DBS), $5.44 \%$ (whole blood) and 5.47\% (plasma), while interbatch precision (\%CV) was below $14.79 \%$ (DBS), $16.12 \%$ (whole blood) and 10.39\% (plasma). Accuracy (\%bias) was below $11.66 \%$ (DBS), $8.49 \%$ (whole blood) and $4.10 \%$ (plasma). All matrix effect and recovery data approximated $100 \%$ and were reproducible. DBS were stable for 324 days at room temperature and for 4 days at $50^{\circ} \mathrm{C}$. Whole blood and plasma samples were stable for 172 days at $-20^{\circ} \mathrm{C}$.

\subsubsection{Impact of DBS-specific parameters on CYP1A2 phenotyping index}

To evaluate the effect of varying hematocrit (Hct) on the concentration of caffeine and paraxanthine, we prepared blood samples with Hct values of 0.20, 0.25, 0.30, 0.43, 0.54 and 0.60 as described before [27]. These were used to prepare low and high quality control samples (QCs) 
( $\mathrm{n}=6$; respectively 0.12 and $8.0 \mu \mathrm{g} / \mathrm{mL}$ for caffeine and 0.06 and $4.0 \mu \mathrm{g} / \mathrm{mL}$ for paraxanthine), from which $25-\mu \mathrm{L}$ DBS were prepared. The influence of blood volume spotted and the impact of punch localization were investigated by preparing low and high QCs in blood with Hct at low $(0.36 \pm 0.007)$, intermediate $(0.43 \pm 0.006)$ and high $(0.50 \pm 0.008)$ Hct. These Hct values were chosen based upon the reference ranges for women and men, which lie at approximately $0.36-$ 0.44 and $0.41-0.50$, respectively [11]. To evaluate the volume effect, different volumes of blood $(7.5,25$ and $50 \mu \mathrm{L})$ were spotted onto the filter paper $(\mathrm{n}=6)$. For the effect of both Hct and volume, the results obtained for $25 \mu \mathrm{L}$ DBS with a Hct of 0.43 were taken as a reference. The impact of punch localization was evaluated by comparing concentrations measured in central versus peripheral punches $(\mathrm{n}=6)$ using an independent samples t-test $(\alpha=0.05)$.

\subsection{Data analysis}

Peak areas were determined using AB SCIEX Analyst 1.5.2 software. Microsoft Excel 2010 and IBM SPSS Statistics 20 were used for statistical evaluation of the data. Bland-Altman plots and Passing-Bablok regression analyses were generated using MedCalc Version 12.7.5.

\section{Results}

\subsection{CYP1A2 phenotyping study}

Seventy-three healthy volunteers (age $21-48$ years) participated in the study, including 50 women and 4 smokers. All participants received a $150 \mathrm{mg}$ oral dose of caffeine. Caffeine and paraxanthine concentrations were determined in capillary DBS obtained 5 minutes before administration and in capillary DBS, venous DBS, whole blood and plasma obtained 6 hours post-administration. Table 1 shows the median concentrations, along with the observed ranges at the 6-hour time point. For 12 volunteers, whole blood samples were not available. Since Perera et al. found that paraxanthine:caffeine ratios were not affected by pre-dose concentrations, whether or not a 24-hour abstinence period was applied [28], the pre-dose concentrations were not taken into account in this study. Bland-Altman comparisons were used to evaluate the data obtained for the different matrices. The mean differences and the limits of agreement (LoAs) are listed in Table 2. Percentage plots were constructed since standard deviations increased with measured concentrations or concentration ratios for most comparisons [29]. Venous whole blood concentrations of caffeine and paraxanthine were on average respectively 15.2 and $16.6 \%$ lower 
than concentrations measured in corresponding plasma samples $(\mathrm{p}<0.001)$. Surprisingly, also a difference between capillary and venous DBS was observed: concentrations of caffeine and paraxanthine in capillary DBS were on average respectively 12.7 and $13.8 \%$ lower than in venous DBS ( $p<0.001)$. There was no significant difference between concentrations in venous DBS and venous whole blood samples ( $\mathrm{p} \geq 0.089$ ), demonstrating the validity of the DBS approach: the entire process of spotting, drying and storing of the samples had no influence on the analytical result. As a result, concentrations in capillary DBS were significantly $(\mathrm{p}<0.001)$ and considerably lower than concentrations in venous plasma samples (on average -31.5 and $-33.1 \%$ for caffeine and paraxanthine, respectively). However, the use of paraxanthine:caffeine molar concentration ratios alleviated both the blood-plasma difference and the capillary-venous difference: mean differences between the different matrices were only -1.6 to $0.6 \%$ (Table 2). In addition, paired samples t-tests showed no significant differences between ratios measured in the different matrices ( $\mathrm{p} \geq 0.053$ ). The differences between measured concentrations in capillary DBS and plasma on the one hand and the equivalence between the phenotyping indices obtained from these matrices on the other hand can readily be deduced from Figure 1, depicting the Passing-Bablok regression analyses of caffeine concentrations (Figure 1a), paraxanthine concentrations (Figure 1b) and paraxanthine:caffeine molar concentration ratios (Figure 1c), as well as the Bland-Altman comparison of these ratios (Figure 1d) in capillary DBS versus those in the reference matrix for CYP1A2 phenotyping, i.e. venous plasma. Bland-Altman plots and Passing-Bablok regression analyses for all comparisons are shown in Supplementary Figures S1S6.

\subsection{DBS-specific parameters}

To evaluate whether -besides capillary-venous differences- other DBS-specific parameters may have an impact on caffeine and paraxanthine quantitation, we varied the Hct, volume spotted and site of punching. Although we and others [20-24] demonstrated that DBS can be used for phenotyping CYP enzymes, no report has evaluated the impact of variation of these parameters on the phenotyping index. First, we did not observe significant differences between caffeine and paraxanthine concentrations measured in discs punched out peripherally versus centrally, irrespective of the Hct (Supplementary Figure S7). Next, we measured caffeine and paraxanthine concentrations (low and high QCs) in DBS, prepared from blood with varying Hct. Figure 2a 
depicts the $\%$ of the normalized sample with Hct 0.43 , being the Hct of the calibrators, for all tested Hct levels. A "Hct effect", i.e. increasingly deviating measured concentrations with increasingly deviating Hct levels, was observed for both compounds. While in the $0.30-0.60 \mathrm{Hct}$ range, measured concentrations were overall within $\pm 15 \%$ of the concentration of the normalized sample, deviations overall exceeded $15 \%$ at the lowest Hct levels (0.20 and 0.25$)$. Importantly, no suchlike influence of the Hct on the paraxanthine:caffeine molar ratio (i.e. the actual phenotyping index) was observed (Figure 2b), indicating that both compounds are subject to a similar Hct effect.

The influence of the blood volume spotted was evaluated by analyzing DBS with three different blood volumes and Hct levels, taking a $25 \mu \mathrm{l}$ DBS with Hct 0.43 as the reference. For both caffeine and paraxanthine, we observed a trend of increasing concentrations with increased blood volumes, although differences from the normalized sample were overall within $\pm 15 \%$ (Figure 2c). Similar to the Hct effect, the paraxanthine:caffeine molar ratio was unaffected by the blood volume (Figure 2d).

\section{Discussion}

Several approaches have been proposed as convenient alternatives for classical plasma-based phenotyping. While for CYP1A2, Perera et al. readily demonstrated the utility of oral fluid for phenotyping [28], we investigated here in-depth the potential of DBS for this purpose. In this study, which is the largest published DBS-based phenotyping study to date, caffeine and its main metabolite paraxanthine were determined in capillary DBS, venous DBS, whole blood and plasma in the context of CYP1A2 phenotyping. Although a proof-of-principle of DBS-based CYP phenotyping is already available [20-24], all published studies had small data sets ( $\leq 16$ ) and did not include a systematic evaluation of DBS-specific parameters. Moreover, with a single exception [23], all studies started from volumetrically applied DBS, an approach that is difficult to sustain when envisaging patient self-sampling at home. In this study, by taking CYP1A2 as an example, the usefulness of a DBS-based phenotyping approach was demonstrated in 73 volunteers using spots prepared by non-experienced individuals in a non-volumetric way. By thoroughly investigating several known challenges associated with DBS analysis, we provide a better insight in the factors underlying the trustworthiness of DBS for phenotyping purposes. 
First of all, significant differences were found between blood and plasma concentrations and between capillary and venous concentrations. As caffeine and most probably also paraxanthine (given their structural similarity) exhibit low binding to plasma proteins and freely enter blood cells without binding to cellular proteins, plasma and blood concentrations should be very similar. Taking into account the fraction of solid constituents in blood, the blood concentration could be expected to lie approximately $15 \%$ lower than the plasma concentration $[9,30]$. The observed differences (15.2 and 16.6\% for caffeine and paraxanthine, respectively) are in line with this expectation. Differences between capillary and venous concentrations have been found for several other small molecules (e.g. piperaquine, paracetamol) [7, 8]. However, lower capillary concentrations of caffeine and paraxanthine compared to venous concentrations, have, to the best of our knowledge, not been described before. Although we cannot fully exclude that anticoagulated blood spotted onto filter paper might exhibit a somewhat different behavior than blood without anticoagulant which is directly applied to the filter paper, we are not aware of evidence supporting this hypothesis. Instead, we consider it more likely that the observed differences between venous and capillary concentrations are caused by as yet incompletely understood physiological factors [31].

In addition to the factors described above, we also evaluated the influence of Hct, blood volume spotted and punch localization. Hct and blood volume had a considerable impact on the concentrations of caffeine and paraxanthine, similar to that described for several other small molecules [32-35]. The range in which the impact of Hct was evaluated, $0.20-0.60$, was considered to be relevant and sufficient, as it covers approximately $99.5 \%$ of a hospital population (including both 'normal' and 'critically ill' patients) [10]. The $7.5-50 \mu \mathrm{L}$ volume range was chosen to represent typical spot sizes resulting from a single drop of blood. Taken the above into account, it can be concluded that, apart from the plasma-blood and the capillary bloodvenous blood difference, also the impact of Hct and volume spotted complicate the interpretation of DBS results. However, we demonstrated that the mean differences between ratios in the different matrices were very limited (-1.6 to $0.6 \%)$ and that the paraxanthine:caffeine molar ratio was unaffected by both Hct and blood volume. The use of metabolite:substrate ratios may therefore offer a strategy to by-pass the impact of these DBS-related issues, demonstrating the usefulness of DBS-based phenotyping approaches. 
Further evidence for the suitability of capillary DBS as a valid alternative for plasma, here considered the reference matrix, in the context of CYP1A2 phenotyping is given by the following: i) the $95 \%$ confidence interval of the average difference between paraxanthine:caffeine ratios in capillary DBS and plasma, obtained by Bland-Altman comparison, contained 0 (Figure 1d), indicating the absence of a consistent bias; ii) PassingBablok regression analysis yielded a good correlation between phenotyping indices from capillary DBS and plasma. As the $95 \%$ confidence intervals of the intercept and the slope contain 0 and 1, respectively (Figure 1c), there were no systematic and proportional differences between the two methods; iii) there was no significant difference between ratios in both matrices, as determined by a paired samples t-test; iv) although no general cut-off points concerning the CYP1A2 phenotype (poor versus rapid metabolizer) are available, we found that the $10 \%$ lowest and highest ratios measured in plasma and capillary DBS corresponded to the same subjects. In this study, the phenotyping index in plasma ranged from 0.090-0.95, which is in line with previous reports $[36,37]$.

\section{Conclusion}

In the largest published DBS-based phenotyping study to date, we demonstrated that quantitation of caffeine and paraxanthine in non-volumetrically applied capillary DBS is not only impacted by Hct and volume spotted, but also significantly differs from venous blood and plasma-based quantitation. However, as the use of the paraxanthine:caffeine molar ratio, i.e. the actual CYP1A2 phenotyping index, alleviates all these issues, we not only demonstrated here that capillary DBS-based CYP1A2 phenotyping is a valid and convenient alternative for the classical plasma-based approach, but, importantly, we also provided an objective basis as to why DBS are an ideal tool for CYP1A2 phenotyping. It needs to be evaluated whether these findings can be generalized for other CYP isoforms, using other CYP substrates. 


\section{Acknowledgements}

The authors wish to thank all volunteers who participated in the phenotyping study.

\section{Conflicts of Interest}

The authors have no conflict of interest to declare. This study was financed by the Laboratory of Toxicology, Ghent University, Belgium. The authors received no additional funding directly related to the content of this study.

\section{References}

1. Demirev PA. Dried blood spots: analysis and applications. Anal Chem. 2013;85(2):77989.

2. Edelbroek PM, van der Heijden J, Stolk LM. Dried blood spot methods in therapeutic drug monitoring: methods, assays, and pitfalls. Ther Drug Monit. 2009;31(3):327-36.

3. Li W, Tse FL. Dried blood spot sampling in combination with LC-MS/MS for quantitative analysis of small molecules. Biomed Chromatogr. 2010;24(1):49-65.

4. Meesters RJ, Hooff GP. State-of-the-art dried blood spot analysis: an overview of recent advances and future trends. Bioanalysis. 2013;5(17):2187-208.

5. Stove CP, Ingels AS, De Kesel PM, Lambert WE. Dried blood spots in toxicology: from the cradle to the grave? Crit Rev Toxicol. 2012;42(3):230-43.

6. Ingels AS, Hertegonne K, Lambert WE, Stove CP. Feasibility of following up gammahydroxybutyric acid concentrations in sodium oxybate $\left(\mathrm{Xyrem}^{\circledR}\right)$-treated narcoleptic patients using dried blood spot sampling at home: an exploratory study. CNS Drugs. 2013;27(3):233-7.

7. Ashley EA, Stepniewska K, Lindegardh N, Annerberg A, Tarning J, McGready R et al. Comparison of plasma, venous and capillary blood levels of piperaquine in patients with uncomplicated falciparum malaria. Eur J Clin Pharmacol. 2010;66(7):705-12.

8. Mohammed BS, Cameron GA, Cameron L, Hawksworth GH, Helms PJ, Mclay JS. Can finger-prick sampling replace venous sampling to determine the pharmacokinetic profile of oral paracetamol? Brit J Clin Pharmacol. 2010;70(1):52-6. 
9. Rowland M, Emmons GT. Use of Dried blood spots in drug development: pharmacokinetic considerations. AAPS Journal. 2010;12(3):290-3.

10. De Kesel PM, Sadones N, Capiau S, Lambert WE, Stove CP. Hemato-critical issues in quantitative analysis of dried blood spots: challenges and solutions. Bioanalysis. 2013;5(16):2023-41.

11. Denniff P, Spooner N. The effect of hematocrit on assay bias when using DBS samples for the quantitative bioanalysis of drugs. Bioanalysis. 2010;2(8):1385-95.

12. O'Mara M, Hudson-Curtis B, Olson K, Yueh Y, Dunn J, Spooner N. The effect of hematocrit and punch location on assay bias during quantitative bioanalysis of dried blood spot samples. Bioanalysis. 2011;3(20):2335-47.

13. Arranz MJ, de Leon J. Pharmacogenetics and pharmacogenomics of schizophrenia: a review of last decade of research. Mol Psychiatry. 2007;12(8):707-47.

14. Magnusson MO, Dahl ML, Cederberg J, Karlsson MO, Sandstrom R. Pharmacodynamics of carbamazepine-mediated induction of CYP3A4, CYP1A2, and Pgp as assessed by probe substrates midazolam, caffeine, and digoxin. Clin Pharmacol Ther. 2008;84(1):5262.

15. Tantcheva-Poor I, Zaigler M, Rietbrock S, Fuhr U. Estimation of cytochrome P-450 CYP1A2 activity in 863 healthy Caucasians using a saliva-based caffeine test. Pharmacogenetics. 1999;9(2):131-44.

16. Zanger UM, Schwab M. Cytochrome P450 enzymes in drug metabolism: regulation of gene expression, enzyme activities, and impact of genetic variation. Pharmacol Ther. 2013;138(1):103-41.

17. Kalow W, Tang BK. The use of caffeine for enzyme assays - a critical appraisal. Clin Pharmacol Ther. 1993;53(5):503-14.

18. Fuhr U, Jetter A, Kirchheiner J. Appropriate phenotyping procedures for drug metabolizing enzymes and transporters in humans and their simultaneous use in the "cocktail" approach. Clin Pharmacol Ther. 2007;81(2):270-83.

19. Perera V, Gross AS, McLachlan AJ. Measurement of CYP1A2 activity: a focus on caffeine as a probe. Curr Drug Metab. 2012;13(5):667-78.

20. de Boer T, Wieling J, Meulman E, Reuvers M, Renkema G, den Daas I et al. Application of dried blood spot sampling combined with LC-MS/MS for genotyping and phenotyping of CYP450 enzymes in healthy volunteers. Biomed Chromatogr. 2011;25(10):1112-23. 
21. Daali Y, Samer C, Deglon J, Thomas A, Chabert J, Rebsamen M et al. Oral flurbiprofen metabolic ratio assessment using a single-point dried blood spot. Clin Pharmacol Ther. 2012;91(3):489-96.

22. Bosilkovska M, Deglon J, Samer C, Walder B, Desmeules J, Staub C et al. Simultaneous LC-MS/MS quantification of P-glycoprotein and cytochrome $\mathrm{P} 450$ probe substrates and their metabolites in DBS and plasma. Bioanalysis. 2014;6(2):151-64.

23. Donzelli M, Derungs A, Serratore MG, Noppen C, Nezic L, Krahenbuhl S et al. The Basel cocktail for simultaneous phenotyping of human cytochrome P450 isoforms in plasma, saliva and dried blood spots. Clin Pharmacokinet. 2014;53(3):271-82.

24. De Kesel PM, Lambert WE, Stove CP. CYP1A2 phenotyping in dried blood spots and microvolumes of whole blood and plasma. Bioanalysis. 2014;accepted.

25. European Medicines Agency. Guideline on bioanalytical method validation. http://www.ema.europa.eu/docs/en_GB/document_library/Scientific_guideline/2011/08/ WC500150010.pdf (Accessed March 2014).

26. U.S. Department of Health and Human Services. Food and Drug Administration. Center for Drug Evaluation and Research. Center for Veterinary Medicine. Draft Guidance for Industry. Bioanalytical Method Validation.

http://www.fda.gov/downloads/drugs/guidancecomplianceregulatoryinformation/guidance s/ucm368107.pdf (Accessed March 2014).

27. Capiau S, Stove VV, Lambert WE, Stove CP. Prediction of the hematocrit of dried blood spots via potassium measurement on a routine clinical chemistry analyzer. Anal Chem. 2013;85(1):404-10.

28. Perera V, Gross AS, Xu H, McLachlan AJ. Pharmacokinetics of caffeine in plasma and saliva, and the influence of caffeine abstinence on CYP1A2 metrics. J Pharm Pharmacol. 2011;63(9):1161-8.

29. Dewitte K, Fierens C, Stockl D, Thienpont LM. Application of the Bland-Altman plot for interpretation of method-comparison studies: a critical investigation of its practice. Clin Chem. 2002;48(5):799-801.

30. Rainey PM. Relation between serum and whole-blood ethanol concentrations. Clin Chem. 1993;39(11):2288-92.

31. Chiou WL. The phenomenon and rationale of marked dependence of drug concentration on blood sampling site. Implications in pharmacokinetics, pharmacodynamics, toxicology and therapeutics (Part II). Clin Pharmacokinet. 1989;17(4):275-90. 
32. Holub M, Tuschl K, Ratschmann R, Strnadova KA, Muhl A, Heinze G et al. Influence of hematocrit and localisation of punch in dried blood spots on levels of amino acids and acylcarnitines measured by tandem mass spectrometry. Clin Chim Acta. 2006;373(12):27-31.

33. Ingels AS, De Paepe P, Anseeuw K, Van Sassenbroeck D, Neels H, Lambert W et al. Dried blood spot punches for confirmation of suspected gamma-hydroxybutyric acid intoxications: validation of an optimized GC-MS procedure. Bioanalysis. 2011;3(20):2271-81.

34. Mess JN, Taillon MP, Cote C, Garofolo F. Dried blood spot on-card derivatization: an alternative form of sample handling to overcome the instability of thiorphan in biological matrix. Biomed Chromatogr. 2012;26(12):1617-24.

35. Vu DH, Koster RA, Alffenaar JW, Brouwers JR, Uges DR. Determination of moxifloxacin in dried blood spots using LC-MS/MS and the impact of the hematocrit and blood volume. J Chromatogr B: Analyt Technol Biomed Life Sci. 2011;879(15-16):106370 .

36. Dobrinas M, Cornuz J, Oneda B, Kohler Serra M, Puhl M, Eap CB. Impact of smoking, smoking cessation, and genetic polymorphisms on CYP1A2 activity and inducibility. Clin Pharmacol Ther. 2011;90(1):117-25.

37. Han XM, Ou-Yang DS, Lu PX, Jiang CH, Shu Y, Chen XP et al. Plasma caffeine metabolite ratio $(17 \mathrm{X} / 137 \mathrm{X})$ in vivo associated with $\mathrm{G}-2964 \mathrm{~A}$ and $\mathrm{C734A}$ polymorphisms of human CYP1A2. Pharmacogenetics. 2001;11(5):429-35. 
Table 1 Caffeine and paraxanthine concentrations in capillary dried blood spots, venous dried blood spots, whole blood and plasma ( $n=73, n=61$ for venous whole blood) obtained 6 hours after administration of a $150 \mathrm{mg}$ oral caffeine dose. The median concentrations together with the observed concentration ranges are listed.

\begin{tabular}{ccccc}
\hline Matrix & \multicolumn{2}{c}{ Caffeine concentration } & \multicolumn{2}{c}{ Paraxanthine concentration } \\
& $\mu \mathrm{g} / \mathrm{mL}$ & $\mathrm{nmol} / \mathrm{mL}$ & $\mu \mathrm{g} / \mathrm{mL}$ & $\mathrm{nmol} / \mathrm{mL}$ \\
\hline $\begin{array}{c}\text { Capillary DBS } \\
\text { Median }\end{array}$ & 1.80 & & & \\
Range & $0.73-3.92$ & $3.76-20.20$ & 0.64 & 3.53 \\
& & & & \\
Venous DBS & & & & \\
Median & 2.13 & 10.99 & 0.73 & 4.03 \\
Range & $0.90-3.95$ & $4.62-20.32$ & $0.28-1.10$ & $1.53-6.09$ \\
& & & \\
Venous blood & & & & \\
Median & & 11.06 & 0.73 & 4.05 \\
Range & $0.78-4.17$ & $4.00-21.49$ & $0.31-1.06$ & $1.70-5.86$ \\
& & & \\
Venous plasma & & & & \\
Median & & 13.17 & 0.90 & 5.00 \\
Range & 2.56 & $5.15-23.56$ & $0.36-1.30$ & $2.00-7.24$ \\
\hline
\end{tabular}


Table 2 Bland-Altman comparisons of concentrations of caffeine and paraxanthine and paraxanthine:caffeine molar concentration ratios measured in venous whole blood, plasma and dried blood spots and capillary dried blood spots $(n=73, n=61$ for comparisons with venous whole blood). The mean differences together with the limits of agreement (LoAs) are listed, with the respective $95 \%$ confidence intervals (values between square brackets).

\begin{tabular}{|c|c|c|c|c|}
\hline Comparators & Statistics & $\begin{array}{c}\text { Caffeine } \\
\text { concentration }\end{array}$ & $\begin{array}{l}\text { Paraxanthine } \\
\text { concentration }\end{array}$ & $\begin{array}{c}\text { Paraxanthine:caffeine } \\
\text { molar ratio }\end{array}$ \\
\hline \multirow{3}{*}{$\begin{array}{l}\text { Venous blood - } \\
\text { venous plasma }\end{array}$} & Upper LoA (\%) & $-3.8[-6.4--1.3]$ & $-6.7[-8.9--4.4]$ & $9.4[6.9-11.8]$ \\
\hline & Mean difference (\%) & $-15.2[-16.7--13.7]$ & $-16.6[-17.8--15.3]$ & $-1.4[-2.8-0.012]$ \\
\hline & Lower LoA (\%) & $-26.5[-29.1--24.0]$ & $-26.5[-28.7--24.2]$ & $-12.1[-14.6--9.7]$ \\
\hline \multirow{3}{*}{$\begin{array}{l}\text { Venous DBS - } \\
\text { venous blood }\end{array}$} & Upper LoA (\%) & $17.0[12.8-21.3]$ & $7.4[5.5-9.3]$ & $16.9[13.2-20.5]$ \\
\hline & Mean difference (\%) & $-1.9[-4.3-0.62]$ & $-1.3[-2.4--0.1]$ & $0.6[-1.5-2.7]$ \\
\hline & Lower LoA (\%) & $-20.7[-25.0--16.5]$ & $-9.9[-11.8--8.0]$ & $-15.7[-19.3--12.0]$ \\
\hline \multirow{3}{*}{$\begin{array}{c}\text { Capillary DBS - } \\
\text { venous DBS }\end{array}$} & Upper LoA (\%) & $18.2[11.9-24.5]$ & $16.5[10.3-22.7]$ & $19.2[15.1-23.3]$ \\
\hline & Mean difference (\%) & $-12.7[-16.4--9.0]$ & $-13.8[-17.4--10.2]$ & $-1.1[-3.5-1.3]$ \\
\hline & Lower LoA (\%) & $-43.6[-49.9--37.3]$ & $-44.1[-50.3--37.9]$ & $-21.4[-25.5--17.2]$ \\
\hline \multirow{3}{*}{$\begin{array}{l}\text { Capillary DBS - } \\
\text { venous plasma }\end{array}$} & Upper LoA (\%) & $-9.8[-14.2--5.4]$ & $-11.3[-15.7--6.8]$ & $13.0[10.0-16.0]$ \\
\hline & Mean difference (\%) & $-31.5[-34.1--28.9]$ & $-33.1[-35.7--30.5]$ & $-1.6[-3.3-0.1]$ \\
\hline & Lower LoA $(\%)$ & $-53.2[-57.7--48.8]$ & $-54.9[-59.3--50.4]$ & $-16.2[-19.2--13.2]$ \\
\hline
\end{tabular}


Figure 1 Passing-Bablok regression analysis of caffeine concentrations (a), paraxanthine concentrations (b), paraxanthine:caffeine molar concentration ratios (c) and Bland-Altman comparison of the paraxanthine:caffeine molar concentration ratios (d) in capillary dried blood spots and venous plasma samples $(n=73)$. For the Passing-Bablok regression analyses, 95\% confidence intervals of slope and intercept are shown between square brackets. In the BlandAltman plot, the mean difference and the limits of agreement (LoA) are displayed, together with the $95 \%$ confidence limits (broken lines).
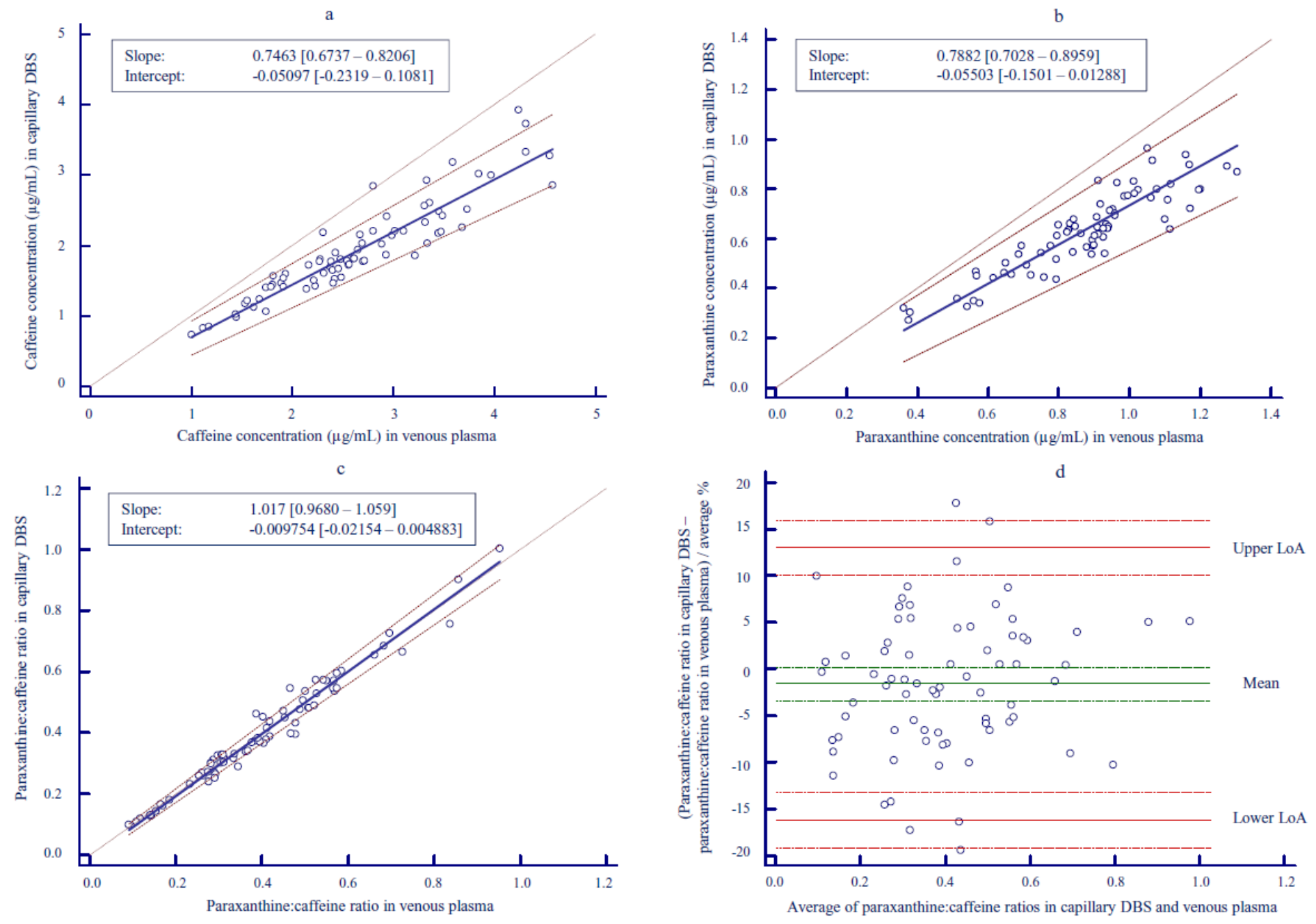
Figure 2 Influence of hematocrit on caffeine and paraxanthine concentrations (a) and paraxanthine:caffeine molar ratios (b). Influence of blood volume spotted on caffeine and paraxanthine concentrations (c) and paraxanthine:caffeine molar ratios (d). Data are presented as percentage of normalized samples with Hct 0.43 and blood volume of $25 \mu L(n=6)$. Dotted lines indicate the $\pm 15 \%$ deviation limits.

a

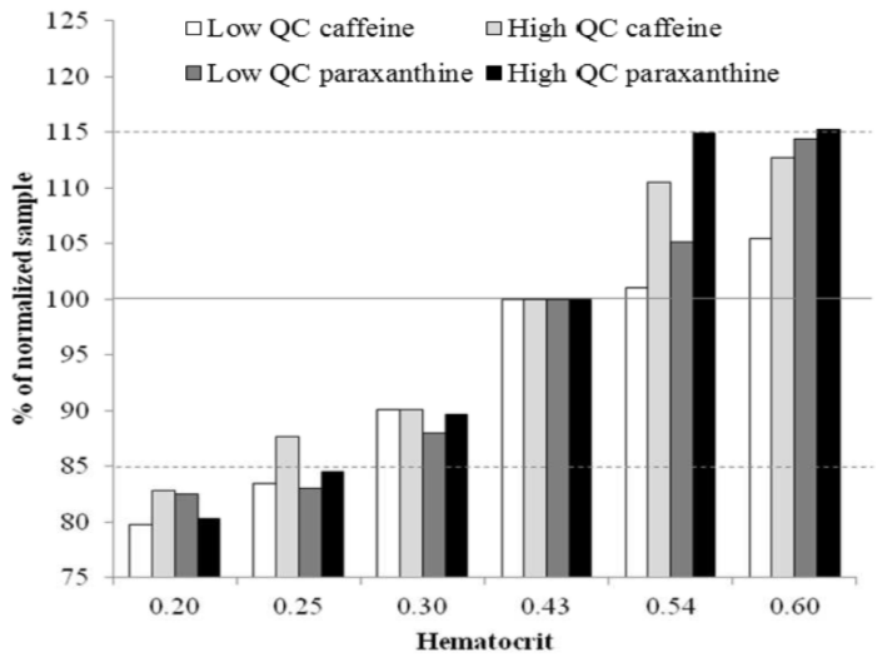

c

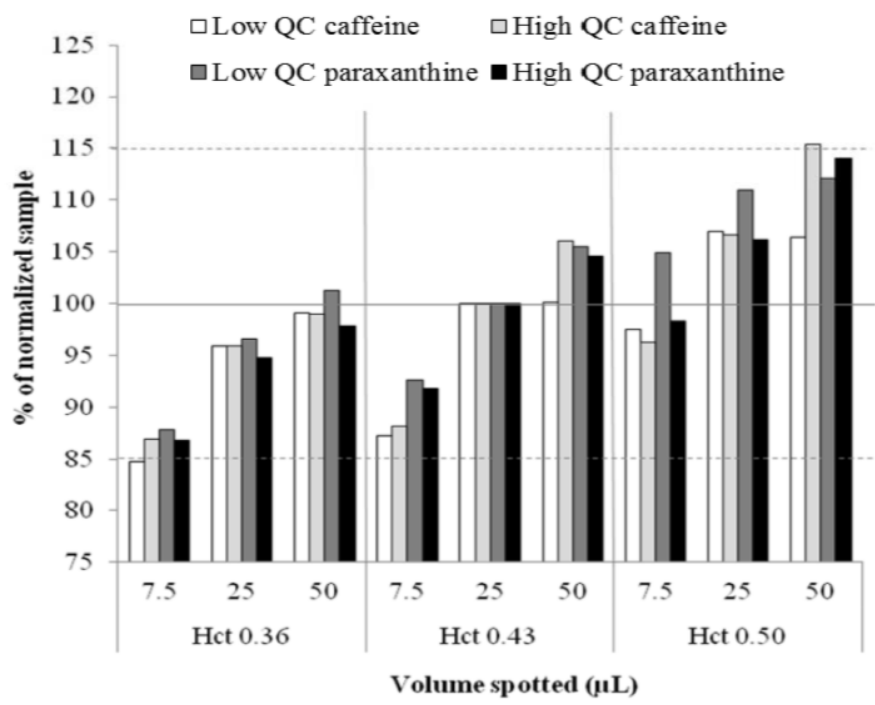

b

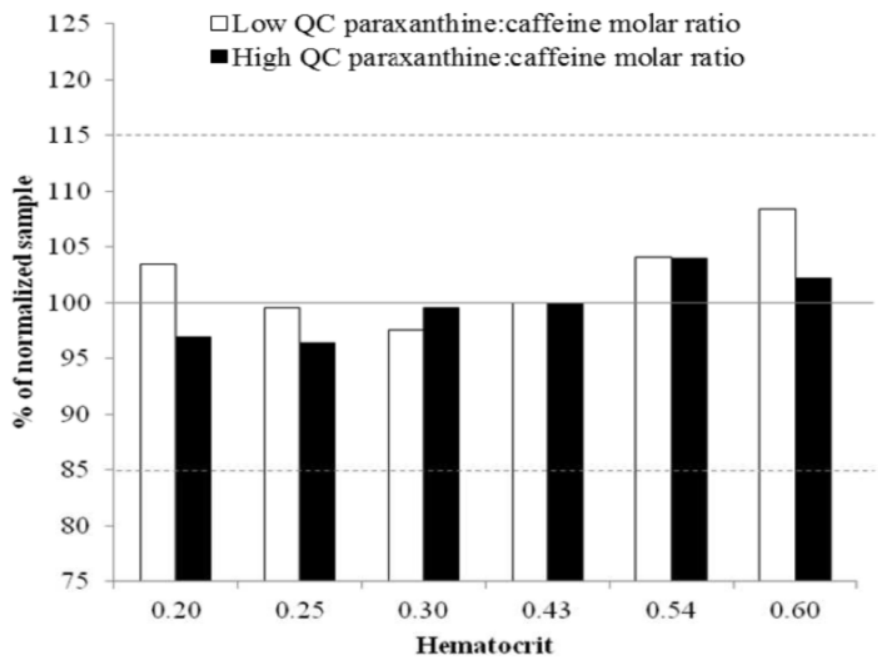

d

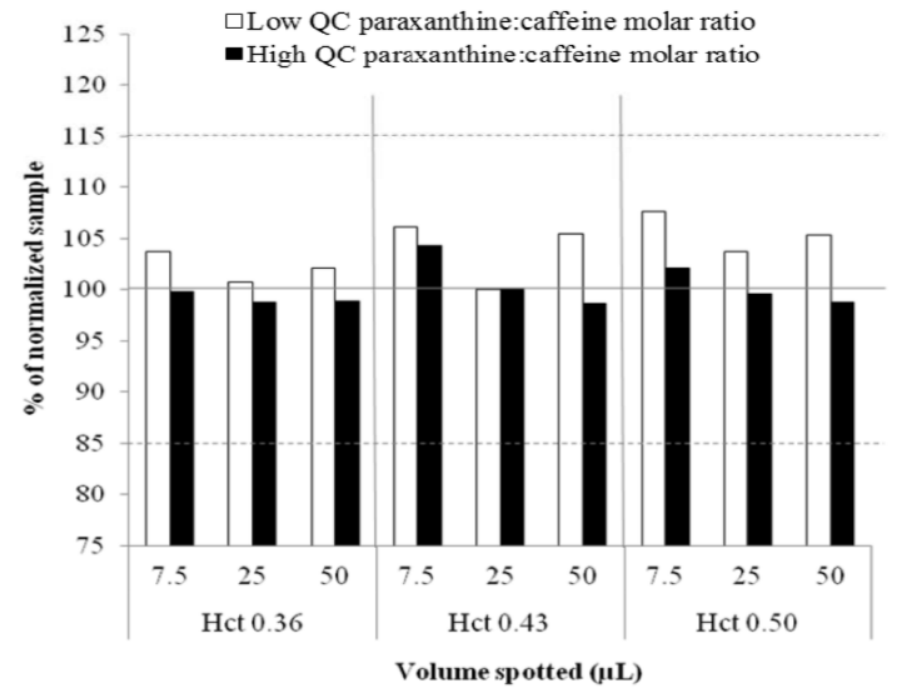

